\title{
RESPONSE OF TUNNEL SCHOTTKY-BARRIER \\ JUNCTIONS TO RADIATION PRESSURE OF FIR \\ LASER RADIATION
}

\author{
S. D. Ganichev, ${ }^{1,3}$ A. Ya. Shul'man, ${ }^{2}$ N. A. Mordovets, ${ }^{2}$ \\ I. N. Kotel'nikov, ${ }^{2}$ and W. Prettl ${ }^{1}$ \\ ${ }^{1}$ Institut für Experimentelle und Angewandte Physik \\ Universität Regensburg, 93040 Regensburg, Germany \\ Institute of Radioenginetring and Electronics of the Russian Academy \\ of Sciences, Moscow 103907, Russia \\ ${ }^{3}$ On leave from A. F. Ioffe Physico-Technical Institute of the \\ Russian Academy of Sciences \\ St. Petersburg 194021, Russia
}

Received May 31, 1996

\section{Abstract}

The response of tunnel Schottky junctions due to plasma reflection of laser radiation has been investigated in the far infrared. The signal was found to be independent of the laser radiation frequency below the plasma edge. At high power levels a nonlinearity of the response "as observed. It is assumed that this nonlinearity is caused by nearzone field effects which lead also to a substantial enhancement of the responsivity.

Key words: far infrared response, tunnel Schottky-barrier, near-zone field effect.

\section{Introduction}

The resistance of tunnel metal-semiconductor junction is very sensitive to the shape of the Schottky-barrier self-consistent potential $[1,2]$. This potential is formed by the charges of ionized impurities located in the depletion layer of the semiconductor, by the charge on the surface states in the semiconductor-metal interface, and by free electron screening. As a result. the equilibrium position 
of the boundary of the free electron plasma in the semiconductor determines the thickness of the Schottky barrier. 'Thus the tumnel resistance is affected by an external force applicd to the clectron plasma. Such a force may be produced by the light pressure arising at total reflection of radiation with frequencies below the plasma edge of the scmiconductor. In previous studies of tunnel Solotikybarrier junctions a fast photoresistive response to pulsed FIR laser radiation has been observed [3]. It was shown that the plasma reflection condition is essential to obtain the fast response. Several other qualitative and semi-quantitative tests and comparisons between $c x-$ perimental data and theoretical model calculations have been carried out [4]. The results allowed to exclude free electron heating due to absorption of radiation as a cause of the observed signals. IIere we present response measurements on $n-C_{x} / \mathrm{As} / \mathrm{Au}$ tunnel junctions in a wide range of intensity for various far-infrared wavelengths yielding more insight in the mechanism of signal generation. At high intensities a superlinear increase of the signal with rising intensity has been obscred for the first tine. This allows to conclude on a radiation ficld enhancement in the near-field zone at the metal-semiconductor interface.

\section{Experimental Technique and Results}

The investigations have been carried out $n$ - GaAs tunnel junctions with plasma reflection minimum near $20 \mathrm{~mm}$ wavelength. The diodes where prepared with semitransparent gold films of about $20 \mathrm{~mm}$ thickness as electrodes having a diameter of $1 \mathrm{~mm}$. On top of each contact an opaque aluminum blind was fixed which left a hole of $0.5 \mathrm{~mm} \mathrm{~m}^{2}$ open area for irradiation. The photoresistive response has been measured as a function of radiation intensity and FIR wavelength. 'The measurements were carried out using a $\mathrm{TEA}-\mathrm{C}^{\circ} \mathrm{O}_{2}$ laser-pumped high power FIR molecular laser. Laser lines with wavelengths $76 \mu \mathrm{m}, 90.5 \mu \mathrm{m}, 152 \mu \mathrm{m}, 385 \mu \mathrm{m}$ and $496 \mu \mathrm{m}$ wore applied within an intensity range of $5 \mathrm{~kW} / \mathrm{cm}^{2}$ up to $1 \mathrm{MW} / \mathrm{cm}^{2}$ and duration of the radiation pulses of the order of $100 \mathrm{~ns}$.

In order to obtain reliable data for the radiation intensity, the geometrical profiles of the spatial distribution of the lascr beam 
near the focus of the optical system were measured by means of the same tunnel junctions under study. Fig. 1 shows the beam

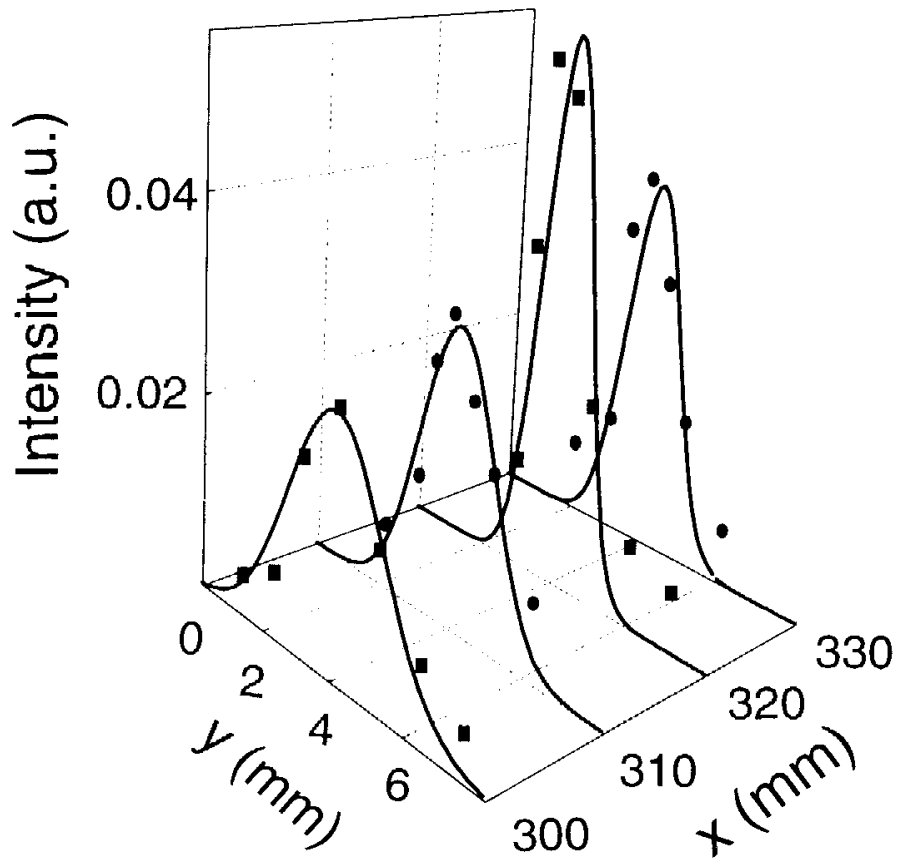

Figure 1: The spatial beam structure $(\lambda=90.5 \mu \mathrm{m})$ for differfnt distances from a parabolic mirror with focal length of $320 \mathrm{~mm}$. The intensity is measured by a tunnel Schottky-barrier junction nGaAs/Au at $T=300 \mathrm{~K}$ with a diameter of $0.5 \mathrm{~mm}$. Solid lines are calculations of the Gaussian beam fitted to experimental data.

profile for $\lambda=90.5 \mu \mathrm{m}$ along the propagation direction $x$ close to the focal spot at $x=320 \mathrm{~mm}$ of a parabolic mirror of $320 \mathrm{~mm}$ focal length. In Fig. 2 the spatial intensity distribution is plotted for $\lambda=250 \mu \mathrm{m}$ in the focal plane of the same parabolic mirror.

In Fig. 3 the tunnel-junction signal is shown as a function of $F I R$ intensity for the various wavelengths. The response depends lincarly on intensity $J$ up to $200-500 \mathrm{~kW} / \mathrm{cm}^{2}$. At higher intensities a deviation of linearity is observed. Fig. 3 also shows that 


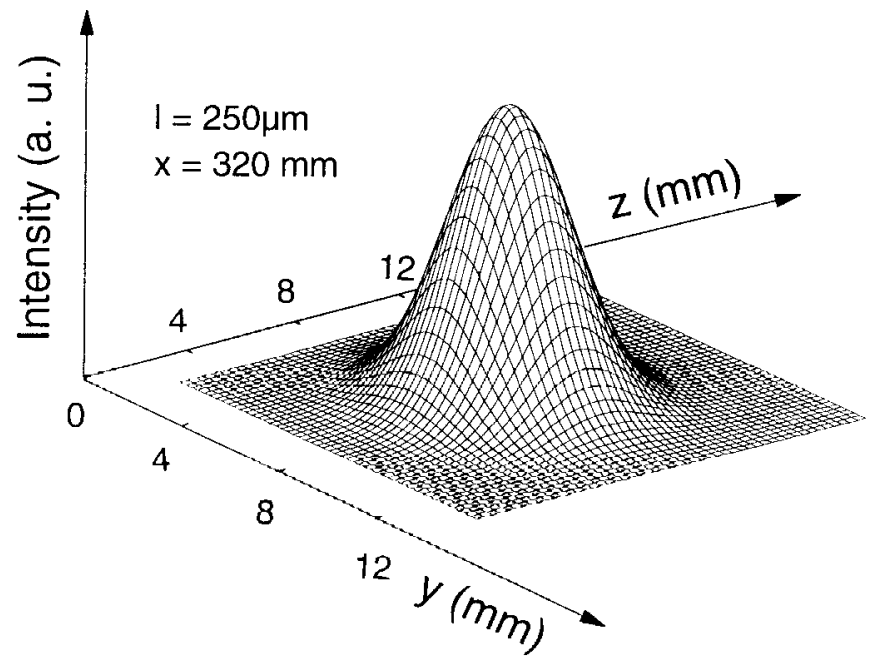

Figure 2: Calculated spatial beam structure in the focal plane of a parabolic mirror with a focal length of $320 \mathrm{~mm}$ for $\lambda=250 \mu \mathrm{m}$. The calculations have been filled to two dimonsional spatially resolved measurements with a tunnel Schotlky-barrier junction n-CiaAs/Au at $T=300 \mathrm{~K}$ having a diameter of $0.5 \mathrm{~mm}$.

the response is independent of the wavelength in a wide spectral range. The response time of the investigated tunnel junctions was about 10 ns. This time is determined by the area of the contacts and may be further decreased by reducing the contact size.

\section{Discussion}

The independence of the signal on wavelength and the linearity of the response at low intensities, are in agreement with theory taking into account the redistribution of free electrons at the plasma boundary caused by the ponderomotive force [4]. This force is assumed to be produced by the evanescent electromagnetic wave during the plasma reflection. The observed magnitude of the signal, however, is about 50 and 16 times higher than given by theoretical estimations for positive and negative bias voltages, respectively. 


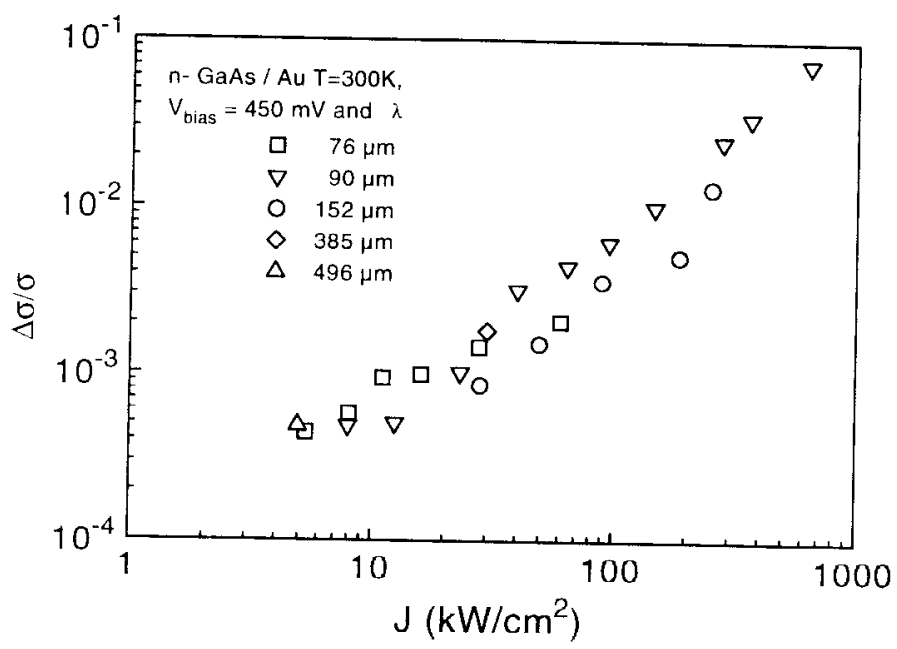

Figure 3: Relative photoconductive signal $\Delta \sigma / \sigma$ as a function of the incident radiation intensity $J$ for different wavelengths. Results are plotted for a tunnel Schottky-barrier junction n-CiaAs/Au at $T-300 \mathrm{~K}$ wilh positive bias voltage $V_{\text {bias }}=150 \mathrm{mV}$.

One of the reasons of this discrepancy could be the local enhancement of the incident wave ficld near the irradiated surface of the semiconductor. Such an enhancement of the radiation field has been observed, for example, in giant Raman scattcring by adatoms on rough metal surfaces [5] where an increase of the scattering inten. sity by a factor of $10^{4}-10^{5}$ was found. Similar effects occur in the case of second harmonic generation by metal surfaces [6]. The source of this enhancement is related to inhomogeneities at the metal surface which favours the generation of surface polaritons or plasmons by the incident radiation even if the angle of incidence is zero (see for instance [7], p 485 and Fig. 4 in [8]). In our case the inhomogeneities may be due to the non-uniformity of the thickness of the semitransparent gold film evaporated on the GaAs surface. It is known, that gold films on GaAs have usually an island structure at thicknesses $\leq 8 \mathrm{~mm}$ and become homogeneous for thicker films. The $A u$ electrodes of the present tunnel junctions with a thickness 
of about $15-20 \mathrm{~nm}$ provide a good conductivity and do not exceed the thickness of the skin layer in the range of radiation frequencies bring of interest here. The films have been shown to be transparent for visible light which was proved by the observation of a barrier photovoltaic effect induced by $H e-N e$ laser radiation. In addition measurements of the free carrier absorption of $10 \mu \mathrm{m}$ radiation transmitted through the $A u$ electrodes revealed that the attenuation of the radiation at this wavelength does not exced 50 percents. Thus for longer wavelength radiation in the far infrared range the transmittance of the $A u$ electrodes should be even higher.

The thickness of the electrodes is very close to that of the transition from the island structure of the deposited film to a homogeneous film. 'Therefore the films may be nonuniform in thickness and transparency for incident radiation. This could lead to an enhancement of the effective field because of the high near-'zone field strength similarly to the case of scattering of radiation by an aperture in a conducting screen (see, for example, relations (13)-(14) in [9]). Such a mechanism may also be responsible lor a surface enhancement of the local ficld which affects the transparency of the tunncl schotuky barricr. On the other hand, surface plasmons must be ruled out because they have a component of the electric field perpendicular to the surface which would enter the Maxwell stress tensor with opposite sign compared to the transverse components. In this case the sign of the signal should be opposite to the observed one (see Fa. (4.5a) iil $[+1])$.

Here we would like to show that the deviation of the response as a function of intensity from linearity at high intensity levels supports the view that near-zone field enhancement of the radiation ficld strength significantly stimulates the tunneling process. The nonlincar dependence $\Delta \sigma / \sigma$ on $J$ allows to determine the absolute magnitude of the electromagnetic field strength in the depletion layer of the Schottky barrier. This will be shown by modifications of the expression for the response of a tunnel junction to the radiation pressure previously derived by us [4].

The tunnel current $I$ is given by:

$$
I \times \int_{0}^{\infty} d E[f(E)-f(E+e V)] \exp (-(i)
$$


where $G$ is in the quasiclassical approximation:

$$
\begin{aligned}
G\left(E, \Phi_{b}, u\right) & \equiv \frac{2(2 m)^{1 / 2}}{\hbar} \int_{x(E, u)}^{0} d x[\Phi(x, u)-E]^{1 / 2}= \\
& =\frac{2(2 m)^{1 / 2}}{\hbar} \int_{E}^{\Phi_{b}} d \Phi \frac{(\Phi-E)^{1 / 2}}{\Phi^{\prime}}= \\
& =\frac{2 \mu}{\hbar \omega_{p}} \int_{\varepsilon}^{W_{b}} d w\left(\frac{w-\varepsilon}{w-2 / 5+K_{e} u / \mu}\right)^{1 / 2}
\end{aligned}
$$

In this equation $f(E)$ is the electron distribution function as a function of the energy $E, m$ is the effective mass of electrons, $\Phi(x)$ is the potential clectron energy in the barricr, $x(E, u)$ is the classical turning point of an electron with energy $E$ in the barrier disturbed by electromagnetic field, $\Phi_{b}=\Phi_{s}+\mu-\epsilon V$ is the band bending at the semiconductor-metal interface, $\Phi_{s}$ is the surface barrier height, $I$ is the hias voltage that is taken to be positive as the electrons tunnel from the semiconductor to metal film, $\Phi^{\prime}(x) \equiv \partial \Phi / \partial x$ is proportional to the static clectric field in the barrier, $\omega_{p}$ is the plasma frequency of the electrons, and $u$ is the high frequency potential [10] produced by the reflected electromagnetic wave in the depletion laver of the Schottky barrier. If the usual Fresnel boundary conditions are applied to the semiconductor-vacuum interface then $u$ is related to the intensity of the incident radiation as:

$$
u \equiv \epsilon^{2}\left|\mathcal{E}_{\infty}\right|^{2} / 4 m \omega^{2}=J / N_{+} c .
$$

where $\mathcal{E}_{\infty}$ is the complex amplitude of the electromagnetic field in the depletion layer, $\omega$ is the radiation frequency, $J$ is the intersity of incident radiation, $N_{\psi}$ is the bulk density of free electrons, and $c$ is the velocity of light in vacuum. The last line in Eq.(2) is written in dimensionless variables $w=\Phi / \mu$ and $\varepsilon=E / \mu$, where $\mu$ is the Fermi energy of the electrons in the semiconductor. Now we can write the expression for the response allowing to study the nonlinear region:

$$
\left.\frac{\Delta \sigma}{\sigma}\right|_{V=\text { const }}=\left.\frac{\Delta I}{I}\right|_{V=\text { const }}=\frac{\eta[I(V, u)-I(V, 0)]}{I(V, 0)},
$$

where $\eta$ is a normalizing factor which has been used to fit the 
calculations to the response measured at small intensities in the linarr regime.

The electric field enhancement at the surface of the semiconductor has been introduced in Eqs. (1) and (2) by assuming a factor $K^{-}$ (see $\mathrm{Eq}$. (2), last line). The results of the calculations of the response for various values $K_{e}$ and $\eta$ are presented in Fig. 4 together with experimental data for the wavelength of $90.5 \mu \mathrm{m}$. It is seen that using a single factor $K_{e}=10^{4}$ permits to describe the nonlinearity of the response for both positive and negative biases. Calculations with other $K_{e}$ shown in Fig. 4 demonstrate that the response as a function of $J$ sensitively depends on this field enhancement factor.

The normalizing factor $\eta$ in Eq. (4) linearly scales the magnilude of the signal $\Delta \sigma / \sigma$ and does not change the intensity dependence. As described above without lield enhancement, i.e. $K_{t}=1$, the observed signal was larger than the alculated one which corresponds to $\eta=50$ for positive bias and $\eta=16$ for negative bias (sor Fig. t). Taking into account the fick enhancement such that the nonlinear intensity dependence is correctly described, normalizing lactors $\eta=1 / 200$ and $\eta=1 / 600$, respectively, are necded to get a cuantitative agreement to the measurements.

Inderd this normalizing factor should reflect the relation betwent the area where the ficld enhancement occurs and the area of the whole Au electrode where the dc tumnel current flows. The differ"nce of $y$ for both biasing conditions may be due to neglecting in the alculation the contribution of the electron states below of the Fermi level to the tunnel current at positive bias (see for more details [4]). Howerer, it is seen from Fig. 4 that the ratio between $\eta$ for positive and negative bias is independent of the enhancement factor $K_{c}$, and therefore this difference is not important for the evaluation of the (nhancement factor.

Due to the fact that the tunneling current rises exponentially with increasing the field enhancement coefficient $K_{e}$, only the part of the contact area, where $K_{p}^{\prime}$ is substantially larger than one, yjelds the major contribution to the response. The effective diameter of this area is thus the total diameter of the electrode multiplied by $\eta^{1 / 2}$. With the above value of $\eta=1 / 600$ for negative bias which is more reliable we find for this diameter the value about $40 \mu \mathrm{m}$. This is somewhat less than but still of the order of the radiation wavelength 


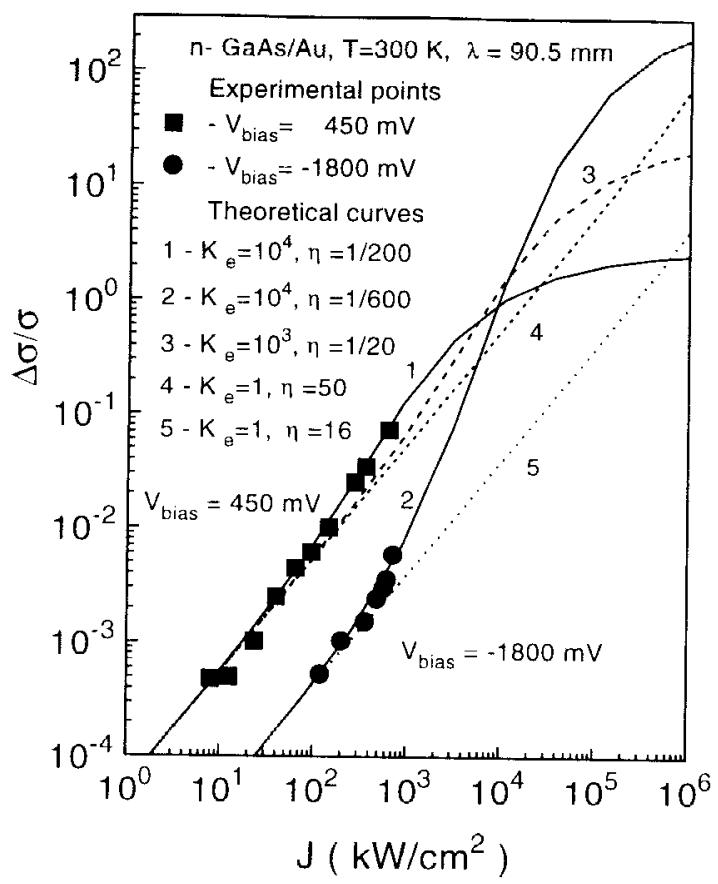

ligure 1: Relatie msponst $\Delta \sigma / \sigma$ as a function of the radiation intemsity f. Siquares and circles are measurements for the positive and the negatier bias noltage bias, respectively. Lines are caleulations "lfer Eq. (4) for different enhancement factors. K.. and normalizing factors. $\eta$. Lines 1. 3, 4 correspond to the positive bias, lines 2 and is to the negation bias. The semiconductor parameters used for culculations are: $\mathrm{V}_{\mathrm{s}}=2 \cdot 10^{18} \mathrm{~cm}^{-3}, \Phi_{s}=0.9 \mathrm{cl}$.

being $90.5 \mathrm{~mm}$ and agrees well with the initial assumption of slge or small aperture effects on the radiation field.

Finally we would like to note that near-field offects may also arise due to a component of the electric ficld normal to the metalsemiconductor interfare. Such a component might appear because of diffraction of the incident electromagnetic ficld on the sffective electroke area. Recently the Bethe problem [11] of the near-field distribution of diffraction at a small aperture was analytically solved for 
the case of normally incident radiation [9]. However, the result shows that the electric ficld perpendicular to the semiconductor surface in the vicinity of the illuminated Schottky junction should be negligible in the region of the Schottky barrier. Nevertheless, a significant enhancement of the transverse electric field should occur near the aperture edge. Such a structure of the field inside the aperture justifies the applications of the expressions derived in [4] for the case of a pure transverse field of the incident wave.

\section{Conclusion}

In summary, the dependence of the tunnel junction response on $\lambda$ and $f$ has been investigated in far-infrared region. The comparison with theoretical calculations indicates the importance of the effects of the enhancement of the radiation field near the semiconductormetal interface. The investigations demonstrate the potential application of tunnel Schotiky diodes as wavelength independent devices to detect FIR laser pulses. The response was found to be linear up to $100 \mathrm{~kW} / \mathrm{cm}^{2}$. The observed near-zone ficld effects show that the sensitivity of detection may considerably be improved compared 10 plain tunneling diodes by proper preparation of the metal electrodes. The time constant of detection may be significantly reduced by down-sizing the area of the junctions up to several $\mu m^{2}$. Subllanosecond time constants seem to be possible. Furthermore by existing technology a large number of junctions may be monolithically integrated yielding a two dimensional array for laser beam profile analysis with high temporal resolution. In addition, a small semiconductor needle with a Schottky contact on its tip may be useful for near field detection as well (see, for instance [12] and references therein).

\section{Acknowledgement}

We wish to thank I.M. Kotelyansky and E. Mirgorodskaya for providing the photolitographic processes. The work has been supported by the International Science Foundation (A. Ya. Sh., N. A. M., and I. N. K., grant MMR000) and by the Deutsche Forschungsge- 
meinschaft (S. D. G., and W. P.).Financial support by the NATO linkage Program (grant HTECH.LG 960398) is gratefully acknowledged.

\section{References}

[1] A.Ya. Shul'man and V.V. Zaitsev, Solid State Comm., 18 (1976) 1623.

[2] I.N. Kotel'nikov; I.L. Beinikhes, and A.Ya. Shul'man, Fiz. Trerd. Tela, 27 (1985) 401 [Sov. Phys. Sol. State. 27 (1985) $246]$.

[3] S.D. Ganichev, I.N. Kotel'nikov, N.A. Mordovets, A.Ya. Shul'man, and I.D. Yaroshetskii, Pis'ma Zh. Exp. Teor. Fiz. 44 (1986) 2:31 [JE'L'P Letters, 44 (1986) 301].

[1] S.I). Ganichev, K.Yu. Glukh, I.N. Kotel'nikov, N.A. Mordovets, A. Ya. Shul'man, and I.D. Yaroshetskii, Zh. Fxp. Teor. Fiz. 102 (1992) 907 [Sov. Phys. JETP, 75 (1992) 495].

[5] ('.Y. C'hen and E. Burstein, Phys. Rev. Lett. 45 (1980) 1287.

[6] J.l. Sipe and (1.I. Stegeman. Nonlinear opticel response of metal surfaces. In Surface Polaritons, ed. by V.M. Agranovich and D.I. Mills, Modern Problems in Condensed Matter Sciences Vol. 1 (North Holland. Amsterdam. 1983) Ch. 15.

[1] E. Burstein. C.Y. Chen, and S. Lundquist. Giant Raman scattering by molccules adsorbed on metals: an overview. In Light Scattering in Solids, Proc. 2nd Joint USA-USSR Symp. on Light Scattering in ('ondensed Matter, ed. by J.L. Birman, II.Z. Cummins, and K.K. Rebane. Plenum, N. Y.. (1979) 479.

[8] A.A. Maradudin, A.R. McGum, R.S. Dummer et al., Optical inleractions at rough surfaces. In Laser Optics of Condensed Matter. Proc.3rd Binational USA-USSR Symp. on Laser Optics of Condensed Matter. Ed. by J.L. Birman, H.Z. ('ummins, and A.A. Kaplyanskii. Plenum, N.-Y., (1987), 127. 
[9] V.V. Klimov and V.S. Letokhov. Optics Comm. 106 (1994) 151.

[10] L.A. Artsimovich and R.Z. Sagdeev, Plasma Physics for Physicists [in Russian], Atomizdat, Moscow, (1979) (Plasma Physik fiir Physiker, Teubner, Stuttgart, 1983).

[11] H.A. Bethe, Phys. Rev. 66 (1944) 163.

$[12]$ F. Depasse and D. Courjon, Optics Comm. 87 (1992) 79. 